\title{
Fog Computing: Terminologies, Architecture and Challenges
}

\author{
Harsimrat Singh, Chetan Marwaha
}

\begin{abstract}
Internet of things (IOT) made the world connected to each other through Internet. These gadgets are important to store data, to exchange data and to collect data from other sources. These devices are not perfectly capable to cooperate with data centers directly based on some parameters such as latency, resource availability, load balancing, scheduling and security. Fog computing (FC) paradigm is introduced to overcome the problems of these parameters. As it cooperate with centralized data centers. This paper presents a survey on Fog computing terminology. Here, the term fog computing has been discussed. Further its architecture, its challenges are highlighted. An overview of further research work related to dynamic job scheduling has been discussed.
\end{abstract}

Keywords: Internet of things, Fog computing, Cloud computing, Latency.

\section{INTRODUCTION}

In modern era, data is increasing day by day. Internet of Things (IOT), Grid Computing and Cloud computing(CC) are some main technologies supporting to generate data and store data. These became the part of our lives and their adoption has risen the use of internet also. As mentioned by Cisco[1] there will be more than 51 billion gadgets connected to Internet by 2020. With Internet, different things such as people, objects, gadgets are connected to communicate with each other at any place and time. Cisco estimated the data generation value will risen up 500+ ZB(zettabytes) [2]. This massive data requires good processing and storage capabilities. Other traditional methods are introduced but not resulted beneficial for handling huge data. Cloud computing came into existence with the integration of parallel, grid and distributed computing. It has various characteristics[3] such as pay-as-per-use, on demand availability, flexible hours service and provides infrastructure, security, platform, software and hardware as a service abbreviated as IaaS, SECaaS, PaaS, SaaS and HaaS respectively[4]. Despite the technology utilization and service provision capability, it has few impediments also. The main problem came is the connectivity and availability between the cloud and the devices[5]. Such availability conditions are set over the

Revised Manuscript Received on July 22, 2019.

* Correspondence Author

Harsimrat Singh*, Dept. of Computer Engineering \& Technology, Guru Nanak Dev University, Amritsar, India. Email:harsimratsingh1996@gmail.com

Chetan Marwaha, Dept. of Computer Engineering \& Technology, Guru Nanak Dev University, Amritsar, India. Email:marwaha.chetan@gmail.com

(c) The Authors. Published by Blue Eyes Intelligence Engineering and Sciences Publication (BEIESP). This is an open access article under the CC BY-NC-ND license (http://creativecommons.org/licenses/by-nc-nd/4.0/)
Internet but are not reasonable for massive set of cloud applications. such applications are fire detection, traffic monitoring and smart grid applications those are generating data in huge amount. And these applications require various components to deploy on multiple clouds and it may lead to high latency. Another problem exists in cloud computing is the processing provision is not near to the user rather than on data centers itself. Cloud provides the high processing speed but at the same time problems like bandwidth usage[6], reliability[7], cost[8], latency[9] and security[10] addressed on cloud computing.

To handle all these problems, Fog computing has been coined that adds the extra layer between clouds and end users. It is the distributed computing solution that connects the various heterogeneous gadgets and brought the cloud data center computation, communication and storage facility at network edges. The connection of end users to the fog nodes through Bluetooth, LTE or 3G/4G results into low data access rate, high performance, and low latency.

This paper focused on survey of fog computing, its basic concepts, their technologies to integrate them with fog computing and its challenges. Second section discusses about the fog computing that contains its definition and overall benefits. Third section explained the more technologies related to fog computing. Fourth section describes the basic architecture of fog systems and next section discusses some important characteristics of fog computing then next section shows the real life applications using fog computing. After that next section enlighten the issues and challenges faced by implementing fog computing. Last section explains the conclusion and future scopes related to this technology and Further section contains references.

\section{FOG COMPUTING}

Fog computing is a technology with different facilities like storage of data, networking and computing services. This technology existence is a decent answer for IOT applications those are sensitive to latency. Inspite of the fact that the term was initially given by CISCO, fog computing has been characterized by numerous analysts from various points of view. In this section we will survey the different definitions given by researchers. CISCO gave its views that fog computing is an expansion of cloud, grid and distributed computing starting from core to edge of network. Fog computing[15] is considered as a situation where countless non-homogenous and decentralized gadgets communicate and collaborate among them and perform different functions like storage and processing.

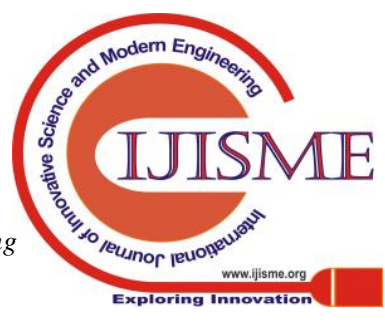


Fog computing is the computing that goes about as middle layer between cloud computing and IOT(Internet of Things) as shown in figure 1. It provides networking, computation and storage facilities. Cisco given the idea of fog computing in 2012 to solve the challenges of IOT gadgets facilities.

IOT gadgets are exceptionally dispersed at the nodes of the centers along with latency-sensitive and storage necessities. Cloud virtual machines are centralized but these are somehow fail to deal with processing speed of IOT devices requests. So, it gives low quality services, high latency and high processing speed as compared to fog computing. Fog computing concept is related to other technologies those are discussed in next section.

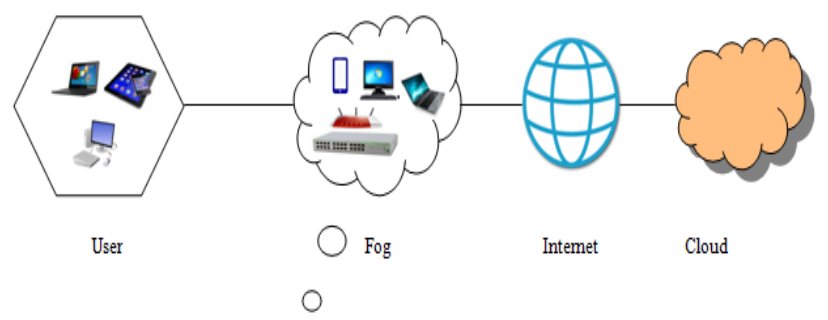

Fig. 1.Fog computing.

\section{RELATED TECHNOLOGIES}

\section{A. Edge Computing}

In current era, the EC(edge computing) in worldwide has increased impressive prevalence in scholastic and mechanical areas. EC fills in as a key empowering agent for some future advancements like 5G, 4G, LTE, Internet of Things (IoT), enlarged real world and node-to-node interchanges by interfacing distributed computing offices and administrations to the end users. Edge computing(EC) comprises another idea in the computing scene. It brings the administration and utilities of distributed computing nearer to the user client and is described using quick handling methods and provide high application response time. As now created web empowered applications, for example, surveillance, computer generated reality and ongoing traffic checking require quick preparing and speedy reaction time $[17,18]$. End clients typically use these working applications on their gadgets those are resource- constrained in manner while the center administration and handling are performed on data centers. Utilizing the facilities provided by cloud using gadgets bring about high latency and mobility related issues. [19,20]. EC satisfies the previously mentioned application necessities by carrying the handling of the edge of the system. The issues of cloud computing can be settled using the three modern technologies such as Cloudlets [21,22],Fog processing [23] and mobile edge computing [24,25]. The European Telecommunications Standards Institute (ETSI) has presented the idea of Mobile Edge computing where mobile clients can use the registering administrations from servers. The fog computing idea has presented by Cisco [26], which empowers the application to run directly at the network edge through billions of smart connected gadgets. Satyanarayanan et al. [27] introduced the idea of cloudlets to solving the delay problem in getting to the cloud to utilizing the PC resources accessible in the nearby arrange. So, also , Mobile edge figuring gives the off-load preparing, application services and capacity near the end clients. With capable highlights of
EC are included low latency, location awareness, proximity to the user and mobility support [28]. These highlights make Edge computing appropriate for various future applications like smart home, virtual reality, industrial automation and data analysis. Edge gadgets like base station, access points, routers [29]. These Edge gadgets go about as an extension that associates the smart cell phones with the cloud. A few studies (S. Yi et al. [30], L.M. Vaquero et al. [31], I. Stojmenovic [32], F. Bonomi et al. [33], T.H. Luan et al. [34], E. Ahmed et al. [35]) have studied different parts of Edge computing domain similar to Mobile Edge computing that focus on explicit application areas. On the other hand, there is no far reaching study has been finished at this point contain all parts of Edge computing include Fog, Mobile-Edge, Mobile Cloud and Cloudlet computing.



Fig. 2.Edge computing.

\section{B. Mobile Edge Computing}

In recent years the equipment of the cell phones keeps on improving, yet at the same time it is viewed as resource constrained. So as to get computational services, for example, Internet of Things (IOT) applications, cell phones as a rule offload these tasks to other incredible computational grids which are in the physical closeness of the offloader [43,44]. A great deal making the mobile clients empowered to encounter the gigantic measure of connecting with applications. On the other hand the immense measure of interest for mobile computing consistently developing, the computing capacity of the cell phones is as yet restricted because of their compact sizes. This expanding request of processing likewise builds the complexities of the computational components [45].

The mobile cloudlet is a believed resource group of cell phones asssociated with one another utilizing the spine network [46]. By giving low-latency access to rich computing resource, cloudlets can drastically improve the presentation of portable applications, by permitting mobile clients offload the applications to adjacent cell phones. These little cloudlets can be associated with different cloudlets to frame a system [47]. Cloudlets can be conveyed in Wide Metropolitan Area Networks (WMANs).

Current cell phones are winding up increasingly incredible and the mobile applications are progressively computation-intensive and delay-delicate, for example, web based gaming [48], extended/virtual reality (ER/VR) [49], picture/video handling APPs [50] and organizing the vehicle frameworks [51]. Applications can regularly present a lot of traffic and computational remaining task at hand, which can conceivably cause the battery channel issue on cell phones [52].

Published By:

Blue Eyes Intelligence Engineering

\& Sciences Publication

(C) Copyright: All rights reserved. 
To manage the resource limitations of cell phone, Mobile Edge Computing has developed as a promising computing worldview. In the Mobile Edge Computing, various little cell base stations (sBSs) with computation and capacity ability [53] are conveyed to develop a system named as the mobile edge network.

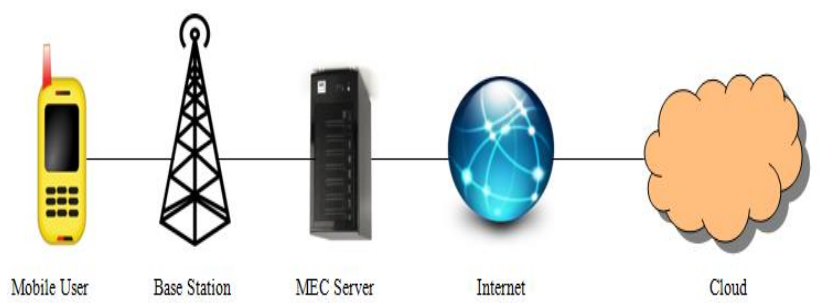

Fig. 3.Mobile Edge computing.

\section{Mobile Cloud Computing}

The expanding number of portable applications and the prevalence of distributed computing, the blend of these two methods that named mobile cloud computing (MCC) pulls in extraordinary consideration recorded by lately [36,37]. MCC is an administration that premits portable clients obliged with assets to adaptively modify handling and putting away capacities by straightforwardly partitioning and offloading the computationally intensive and capacity requesting occupations on customary cloud assets by giving everywhere remote access [37]. In the previous portable figuring worldview, there are a few problems such as frequent disconnections, mobility and resource scarcity. With the help of mobile cloud computing, the previously mentioned issues can be tended to and the users of mobile can accomplish consistent access and handover for administrations, since portable applications are are executed on resource providers outer to the cell phone.

The expanding utilization of mobile computing is deal by the examination by Researcher, that further expresses the buyer and endeavor showcase for cloud based mobile applications is relied upon to ascend to $\$ 9.6$ billion by 2017 [38]. In latest years, services focused at gadgets have begun getting to be many services in different classifications methods such as entertainment, social networking, health, games and news. The ubiquity of these are apparent by perusing through mobile application download focuses such as Apples's iTunes or Nokia's Ovi suite. The explanation behind this mobile computing can give an device to the user when and where it is necessity regardless of end users development, further provided area independence. Undoubtedly, mobility is one of the uniqueness of an avoidable figuring condition where the client user can proceed with the development. Be that with mobility comes its natural problems like asset scarceness, low connectivity and finite energy as sketched out by author of [39]. These deals with the issue of executing number of helpful projects those further use by clients and make an invasive environment. As per Tim O'Reilly the future administrations has some points that react progressively to data gave either by their user or by non-human sensors [40].

Thinking about the trends in cell phone architecture and batteries architecture, it is improbable that these problems will be solved later on. This is the fact that to be discussed, not simply a provisional technological deficiency but rather characteristic for mobility [41], and a boundary that should be defeated so as to understand the maximum capacity of mobile computing. As of late, this issue has been tended to by specialists however cloud computing. Cloud computing can be characterized as the aggregation of computing as an utility and SaaS where the delivery of applications as services over the internet[42].

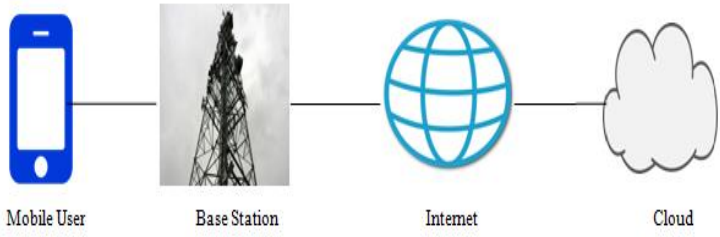

Fig. 4.Mobile Edge computing.

\section{ARCHITECTURE OF FOG SYSTEMS}

Fog computing is a worldview which broadens the distributed computing and cloud computing. It provides storage, network, controlling and many more services at the edge of network. But every platform is somehow different from one another, that can be basis on architecture. The current section elaborates the architecture of fog computing. There are different levels in fog computing layered architecture[16] . The architecture of fog system is the hot research topic. Most of the researchers have worked on it but all the derived architectures follow the basics of layered architecture discussed below. The layers of fog computing are:

A. End Layer: This layer is nearest to the user and physical condition. It comprises of different IOT gadgets such as cell phones, smart readers and smart phones etc. However the mobile phones and vehicles have sensing units, those are responsible for detecting the information and transmitting data to the above layers.

B. Fog Middle Layer: This layer is the middle layer of fog systems. It consists of number of fog nodes, base stations, servers and various access points. These fog nodes are distributed, those lies between cloud and users. The end layer gadgets are associated with fog hubs to acquire services. Those have ability to transmit, compute and store the detected information. This layer is sometimes complicated for latency related applications and real time applications. The nodes in this layer communicate with cloud data centers by using IP addresses.

C. Cloud Top Layer: This layer is high level layer that contains superior servers and high storage devices those provide different services like Gmail these have processing and capacity ability to help for broad calculation.

\section{CHARACTERISTICS OF FOG COMPUTING}

Fog computing plays good role for storage and latency sensitive applications. Fog computing capable of all type of broad computation, communication on edge devices. According to [13] [14]fog computing has characteristics based on different parameters those are summarized as follows:

Published By:

Blue Eyes Intelligence Engineering \& Sciences Publication 
Table- I: characteristics of fog computing based on various parameters

\begin{tabular}{|c|c|}
\hline Parameters & Description \\
\hline $\begin{array}{l}\text { Location awareness, } \\
\text { latency sensitivity }\end{array}$ & $\begin{array}{l}\text { Usage of fog supports location } \\
\text { awareness and sensitivity of latency and } \\
\text { it helps to reduce the time while } \\
\text { processing the data of end devices. It } \\
\text { can be deployed in different location. } \\
\text { Hence, it's work is to enhance the speed } \\
\text { so that message can be conveyed within } \\
\text { few seconds. }\end{array}$ \\
\hline Scalability & $\begin{array}{l}\text { Our ambient is monitored by large-scale } \\
\text { sensor networks. Distribution of } \\
\text { computing and storage resources that is } \\
\text { provided by fog can work with such } \\
\text { large-scale end gadgets. }\end{array}$ \\
\hline $\begin{array}{l}\text { Distribution of } \\
\text { geographical area }\end{array}$ & $\begin{array}{l}\text { In vary to the data center cloud, fog that } \\
\text { provides services, facilities and } \\
\text { applications which can be allocated and } \\
\text { can be sent anywhere. }\end{array}$ \\
\hline Mobility & $\begin{array}{l}\text { Fog applications has the ability to } \\
\text { connect directly to mobile devices and it } \\
\text { enables mobility methods, such as } \\
\text { location ID separation protocol (LISP) } \\
\text { that needs a distributed directory } \\
\text { system. }\end{array}$ \\
\hline Real-time interactions & $\begin{array}{l}\text { Real-time interactions between fog } \\
\text { nodes are provided fog computing } \\
\text { applications instead of the batch } \\
\text { processing employed in the cloud. }\end{array}$ \\
\hline Heterogeneity & $\begin{array}{l}\text { Fog end nodes are available in different } \\
\text { forms, as these are proposed by different } \\
\text { manufactures and are deployed. } \\
\text { According to their platforms because it } \\
\text { has the ability to work on various } \\
\text { platforms. }\end{array}$ \\
\hline Interoperability & $\begin{array}{l}\text { Different services are provided by fog } \\
\text { components that work with different } \\
\text { domains. }\end{array}$ \\
\hline $\begin{array}{l}\text { Support for on-line } \\
\text { analytics and } \\
\text { interplay with the } \\
\text { cloud }\end{array}$ & $\begin{array}{l}\text { Fog plays an important role in } \\
\text { processing of the data. Fog helps to } \\
\text { reduce the time and in the absorption of } \\
\text { the data that's why it is placed between } \\
\text { cloud and end user devices. }\end{array}$ \\
\hline
\end{tabular}

\section{APPLICATIONS OR SERVICES}

The fog computing has an expansive range of utilization in all fields. Bonomi and Flavio et. al [11] have discussed fog computing applications in different areas such as vehicles, smart cities, grids and wireless sensor. [12] have discussed the applications and issues in expanded reality, mobility big data analysis and content conveyance. This section discusses the various applications for fog computing:

\section{A. Home Appliances}

With the use of IOT, an increasing number of savvy gadgets and sensors are associated at home. But the products and gadgets from different merchants are difficult to become compatible. Some of the assignments require high computation, reliability and storage and then fog computing helps to tackle these issues. Fog computing is very helpful to run home appliances, to run home security applications and door lock applications. When the fog layer is introduced in the system, each sensor node works as client and server side works on Virtual machines. Advanced algorithms work behind it. For an example: A sensor detects the movement of person in the room and video analysis also been performed to detect the correct person has been entered in the room or false alarm will be called.

\section{B. Vehicles Management on Roads}

For intelligent transportation service VANET(Vehicular Ad-hoc Network) has been considered. It guarantee traffic efficiency, driving well being and comfort by exchanging important data, they support different portable devices extending from sharing applications to data spreading services such as from advertisement or entertainment to emergency operations in hospitals. During the couple of years, with the rise of cloud computing, 4G, 5G and various applications developed dramatically. As a result of this pattern, an eminent issue shows up, to be specific in case of computation and communication. The augmented reality procedures, all arrangement require high level of calculations and computation.

\section{Better Living Environment}

In this era, better living styles have various facilities like real time interactive applications, location based various services and usage of smart objects. Smart objects are universally distributed and these faces the issue of latency between different devices and data centers. So fog computing helps to act as interface or provide all the computation capabilities locally those scale down the latency and increased the performance. As the above layered architecture also helpful to provide better environment as compared to direct cloud to device communication.

\section{Combination with IOT and Cloud}

As the rise of devices in billions has made difficult to deal with data efficiently. So IOT and cloud combined with fog to provide the man agent for growing data and devices. Including this, it also provide resource provision, administration closure and many more. Fog computing lies in middle of cloud and IOT. The main advantage of this combination can be attain in industry as Industrial IOT where the machines and different sensors and actuators are utilized in the system.

\section{ISSUES AND CHALLENGES}

In this section, we will discuss and identify issues in context of various parameters based on fog computing. These leads to future research work.IOT.

\section{A. Servers}

Fog servers are geo-dispersed and are conveyed at common places such as parks, streets and many more. As light-weight cloud data servers, these fog servers are virtualized and equipped with capacity, storage and organizing offices. There are numerous works those considered fog servers as practical segment of fog computing. some the papers[53][54] gave different names to fog servers and some papers[55][56] discusses the functionality of fog computing.






\section{B. Devices}

Gadgets like switches, plugs, set top boxes can go about as potential foundation for fog computing. In some work, the systems administration gadgets are structured with various framework such as high quality processor, memory and programming platform. Various networking devices like smart hubs, gateways considered as nodes of fog systems[57].

\section{Cloudlets}

These are considered as small scale clouds and situated at the middle layer of gadget. cloudlets[58] are considered as fog nodes those are virtualized and manage huge number of end gadgets at the same time. The disadvantages of centralized computing is significant in fog computing that resist to support IOT.

\section{Base Stations}

Base stations[59] are significant segments in portable and remote systems for consistent correspondence and information handling. In this work, the old base stations are furnished with computation and calculation capabilities.

\section{E. Data Management}

Data management is one of the major issue of fog computing. It is basically required for productive execution. Different services like data analysis and policies for allocation of resources mainly focused on data management. Further low latency accumulation of data generated from portable devices is considered for data management. As storage devices are not so worth to store data, fog computing data management policies[60][61] provide abilities to manage and store the data in organized way.

\section{F. Job scheduling}

It is the major issue that provide the method to handle the job requests of fog computing. The solution for this provide high performance, efficient resource provision and less execution time. For scheduling the tasks and jobs various algorithms such as heuristics, round robin and many can be preferred. [63] this paper worked as heuristic method for scheduling the tasks and jobs.

\section{G. Energy}

This is the high priority issue considered in fog computing. The main issue is energy and latency trade off in various phases are major issue of fog and cloud computing. Various issues and challenges are highlighted as carbon outflow pace of different nodes, energy features of transmission mediums, battery lifetime and many more. [62]

\section{H. Cost}

The cost issue is the common challenge in every computing that cost can be execution, deployment or networking cost, data fetching and uploading cost. Most of the cases the sharing of data and uploading of the cost considered in network phase, the challenge solution can provide efficient provisioning of data and resources.

\section{CONCLUSION AND WORK}

Fog computing is high potential processing model whose significance is increasing because of quick improvement of IOT, cloud computing and Internet. As utilizing topographically system edge gadgets, the fog paradigm pushes an ever increasing number of applications from cloud servers to user end. It incredibly decreases the information move time and measure of system transmission and hence results in fulfill the needs of applications sensitive to latency. In this paper, we focuses on introductory part of fog computing, its applications, challenges and issues. We discusses the different layers contains in fog systems, its characteristics and its comparison with cloud computing. Further this paper elaborates the new challenges, the main area to discuss is job scheduling at fog nodes using different algorithms. Most of the researchers focused on static scheduling algorithm. But with the mobility of devices, dynamic scheduling with suitable load balancing is required. So, more research areas are introduced, those will rise the future scholarly world of organizations.

\section{REFERENCES}

1. D. Evans, The internet of things: How the next evolution of the internet is changing everything, CISCO White Paper 1 (2011) $1\{11$.

2. Cisco global cloud index: Forecast and methodology, 2014-2019 white paper.

3. L. M. Vaquero, L. Rodero-Merino, J. Caceres, and M. Lindner, "A Break in the Clouds: Towards a Cloud Definition," SIGCOMM Comput Commun Rev, vol. 39, no. 1, pp. 50-55, Dec. 2008.

4. Q. Zhang, L. Cheng, and R. Boutaba, "Cloud Computing: State-of-the-art and Research Challenges," J. Internet Serv. Appl., vol. 1, no. 1, pp. 7-18, May 2010.

5. Dillon, Tharam, Chen Wu, and Elizabeth Chang. "Cloud computing: issues and challenges." In 2010 24th IEEE international conference on advanced information networking and applications, pp. 27-33. Ieee, 2010.

6. Popa, Lucian, Praveen Yalagandula, Sujata Banerjee, Jeffrey C Mogul, Yoshio Turner, and Jose Renato Santos. "Elasticswitch: Practical work-conserving bandwidth guarantees for cloud computing." In ACM SIGCOMM Computer Communication Review, vol. 43, no. 4, pp. 351-362. ACM, 2013.

7. Bauer, Eric, and Randee Adams. Reliability and availability of cloud computing. John Wiley \& Sons, 2012.

8. Zhang, Yang, Dusit Niyato, and Ping Wang. "Offloading in mobile cloudlet systems with intermittent connectivity." IEEE Transactions on Mobile Computing 14, no. 12 (2015): 2516-2529.

9. Chen, Min, Yixue Hao, Yong Li, Chin-Feng Lai, and Di Wu. "On the computation offloading at ad hoc cloudlet: architecture and service modes." IEEE Communications Magazine 53, no. 6 (2015): 18-24.

10. Wang, Cong, Qian Wang, Kui Ren, and Wenjing Lou. "Privacy-preserving public auditing for data storage security in cloud computing." In 2010 proceedings ieee infocom, pp. 1-9. Ieee, 2010.

11. Bonomi, Flavio, Rodolfo Milito, Jiang Zhu, and Sateesh Addepalli. "Fog computing and its role in the internet of things." In Proceedings of the first edition of the MCC workshop on Mobile cloud computing, pp. 13-16. ACM, 2012.

12. S. Yi, C. Li, and Q. Li, "A survey of fog computing: Concepts, applications and issues," in Proceedings of the 2015 Workshop on Mobile Big Data. ACM, 2015.

13. Ai, Y.; Peng, M.; Zhang, K. Edge cloud computing technologies for internet of things: A primer.Digit. Commun. Netw. 2017, in press.

14. Yi, S.; Hao, Z.; Qin, Z.; Li, Q. Fog computing: Platform and applications. In Proceedings of the 3rdWorkshop.

15. L. M. Vaquero and L. Rodero-Merino. Finding your way in the fog: Towards a comprehensive de_nition of fog computing. ACM SIGCOMM Computer Communication Review, 2014.

16. Hu, Pengfei, Sahraoui Dhelim, Huansheng Ning, and Tie Qiu. "Survey on fog computing: architecture, key technologies, applications and open issues." Journal of network and computer applications 98 (2017): $27-42$.

17. N. Hassan, S. Gillani, E. Ahmed, I. Yaqoob, M. Imran, The role of edge computing in internet of things, IEEE Communications Magazine (99) (2018) 1-6.




18. M. Liu, F. R. Yu, Y. Teng, V. C. Leung, M. Song, Distributed resource allocation in blockchain-based video streaming systems with mobile edge computing, IEEE Transactionson Wireless Communications 18 (1) (2019) 695-708.

19. E. Ahmed, A. Akhunzada, M. Whaiduzzaman, A. Gani, S. H. Ab Hamid, R. Buyya, Networkcentric performance analysis of runtime application migration in mobile cloud computing, Simulation Modelling Practice and Theory 50 (2015) 42-56.

20. P. Pace, G. Aloi, R. Gravina, G. Caliciuri, G. Fortino, A. Liotta, An edge-based architecture to support efficient applications for healthcare industry 4.0, IEEE Transactions on Industrial Informatics 15 (1) (2019) 481-489.

21. U. Shaukat, E. Ahmed, Z. Anwar, F. Xia, Cloudlet deployment in local wireless networks: Motivation, architectures, applications, and open challenges, Journal of Network and Computer Applications 62 (2016) 18-40.

22. I. Yaqoob, E. Ahmed, A. Gani, S. Mokhtar, M. Imran, S. Guizani, Mobile ad hoc cloud: A survey, Wireless Communications and Mobile Computing 16 (16) (2016) 2572-2589.

23. W. Bao, D. Yuan, Z. Yang, S.Wang, W. Li, B. B. Zhou, A. Y. Zomaya, Follow me fog: Toward seamless handover timing schemes in a fog computing environment, IEEE Communications Magazine 55 (11) (2017) 72-78. doi:10.1109/MCOM.2017.1700363.

24. E. Ahmed, M. H. Rehmani, Mobile edge computing: opportunities, solutions, and challenges, Future Generation Computer Systems 70 (2017) 59-63.

25. Y. Jararweh, A. Doulat, O. AlQudah, E. Ahmed, M. Al-Ayyoub, E. Benkhelifa, The future of mobile cloud computing: integrating cloudlets and mobile edge computing, in: Telecommunications (ICT), 2016 23rd International Conference on, IEEE, 2016, pp. 1-5.

26. Cisco fog computing solutions: Unleash the power of the internet of things, available

https://www.cisco.com/c/dam/en_us/solutions/trends/iot/docs/computi ng-solutions.pdf, 2015 (Accessed on 23 July 2018).31

27. M. Satyanarayanan, P. Bahl, R. Caceres, N. Davies, The case for VM-based cloudlets in mobile computing, IEEE Pervasive Computing 8 (4) (2009) 14-23. doi:10.1109/mprv.2009.82.

28. A. Ahmed, E. Ahmed, A survey on mobile edge computing, in: 10th International Conference on Intelligent Systems and Control (ISCO), 2016, pp. 1-8. doi:10.1109/ISCO.2016.7727082.

29. Z. Zhang, W. Zhang, F. Tseng, Satellite mobile edge computing: Improving qos of high-speed satellite-terrestrial networks using edge computing techniques, IEEE Network 33 (1) (2019) 70-76. doi:10.1109/MNET.2018.1800172.

30. S. Yi, C. Li, Q. Li, A survey of fog computing: concepts, applications and issues, in: Proceedings of the 2015 workshop on mobile big data, ACM, 2015, pp. 37-42.

31. L. M. Vaquero, L. Rodero-Merino, Finding your way in the fog: Towards a comprehensive definition of fog computing, ACM SIGCOMM Computer Communication Review 44 (5) (2014) 27-32.

32. I. Stojmenovic, S. Wen, The fog computing paradigm: Scenarios and security issues, in: Computer Science and Information Systems (FedCSIS), 2014 Federated Conference on, IEEE, 2014, pp. 1-8.

33. F. Bonomi, R. Milito, J. Zhu, S. Addepalli, Fog computing and its role in the internet of things, in: Proceedings of the first edition of the MCC workshop on Mobile cloud computing, ACM, 2012, pp. 13-16.

34. T. H. Luan, L. Gao, Z. Li, Y. Xiang, G. Wei, L. Sun, Fog computing: Focusing on mobile users at the edgearXiv:1502.01815v3.

35. E. Ahmed, A. Ahmed, I. Yaqoob, J. Shuja, A. Gani, M. Imran, M. Shoaib, Bringing computation closer toward the user network: Is edge computing the solution?, IEEE Communications Magazine 55 (11) (2017) 138-144.

36. N Fernando, SW Loke, W Rahayu, Mobile cloud computing: a survey[J]. Future Generation Comput. Syst 29(1), 84-106 (2013).

37. AN Khan, MLMKiah, SU Khan, SAMadani, Towards securemobile cloud computing: a survey[J]. Future Generation Comput. Syst 29(5), 1278-1299 (2013)

38. S. Perez, Mobile cloud computing: $\$ 9.5$ billion by 2014 http://exoplanet.eu/catalog.php, 2010

39. M. Satyanarayanan, Fundamental challenges in mobile computing, in: Proceedings of the Fifteenth Annual ACM Symposium on Principles ofDistributed Computing, PODC'96, ACM, New York, NY, USA, 1996, pp. 1-7.

40. L. Siegele, Let it rise: a special report on corporate it, http://www.economist.com/node/12411882, 2008.

41. M. Armbrust, A. Fox, R. Griffith, A. Joseph, R. Katz, A. Konwinski, G. Lee, D.Patterson, A. Rabkin, I. Stoica, Above the clouds: a Berkeley view of cloud computing, Technical Report UCB/EECS-2009-28, 2009.
42. M. Jia, J. Cao, W. Liang, Optimal cloudlet placement and user to cloudlet allocation in wireless metropolitan area networks, IEEE Trans. Cloud Comput. 5 (4) (2017) 725-737.

43. R. Shit, S. Sharma, D. Puthal, A.Y. Zomaya, Location of Things (LoT) A review and taxonomy of sensors localization in IoT infrastructure, IEEE Commun. Surv. Tutorials (2018) http://dx.doi.org/10.1109/COMST.2018.2798591.

44. M. Satyanarayanan, G. Lewis, E. Morris, S. Simanta, J. Boleng, H Kiryong, The role of cloudlets in hostile environments, Proc. IEEE Pervasive Comput. 12 (4) (2013) 40-49.

45. L. Yujin, Can mobile cloudlets support mobile applications? in: The Proc of IEEE INFOCOM, 2014, pp. 1060-1068.

46. HAProxy, HAProxy - the Reliable, High Performance TCP/HTTP Load Balancer, 2017. http://www.haproxy.org/.

47. D. Meilander, F. Glinka, S. Gorlatch, L. Lin, W. Zhang, X. Liao, Bringing mobile online games to clouds, in: Computer Communications Workshops, INFOCOM WKSHPS, 2014 IEEE Conference on, IEEE, 2014, pp. 340-345.

48. W. Barfield, Fundamentals of Wearable Computers and Augmented Reality, CRC Press, 2015.

49. P. Mach, Z. Becvar, Mobile edge computing: A survey on architecture and computation offloading, IEEE Commun. Surv. Tutor. (2017).

50. Y. Wu, G. Min, L.T. Yang, Performance analysis of hybrid wireless networks under bursty and correlated traffic, IEEE Trans. Veh. Technol. 62 (1) (2013) 449-454.

51. X. Ma, P. Huang, X. Jin, P. Wang, S. Park, D. Shen, Y. Zhou, L.K Saul, G.M. Voelker, eDoctor: Automatically diagnosing abnormal battery drain issues on smartphones, in: NSDI, Vol. 13, 2013, pp $57-70$

52. Lee, W., K. Nam, H.G. Roh, S.H. Kim. 2016. A gateway based fog computing architecture for wireless sensors and actuator networks. In 18th International Conference on Advanced Communication Technology (ICACT), IEEE, 210-213.

53. Aazam, M., and E.N. Huh. 2015. Fog computing micro datacenter based dynamic resource estimation and pricing model for iot. In IEEE 29th International Conference on Advanced Information Networking and Applications. (March 2015), 687-694.

54. Zhu, J., D.S. Chan, M.S. Prabhu, P. Natarajan, H. Hu, F. Bonomi. 2013. Improving web sites performance using edge servers in fog computing architecture. In Service Oriented System Engineering (SOSE), 2013 IEEE 7th International Symposium on, (March 2013) 320-323.

55. Zeng, D., L. Gu, S. Guo, Z. Cheng, and S. Yu. 2016. Joint optimization of task scheduling and image placement in fog computing supported software-defined embedded system. IEEETransactions on Computers PP(99): 1-1.

56. Aazam, M., and E.N. Huh. 2014. Fog computing and smart gateway based communication for cloud of things. In Future Internet of Things and Cloud (FiCloud), International Conference on IEEE (2014), 464-470.

57. Dsouza, C., G.J. Ahn, and M. Taguinod.2014. Policy-driven security management for fog computing: Preliminary framework and a case study. In: IEEE 15th International Conferenceon Information Reuse and Integration (IRI), (Aug 2014), 16-23.

58. Yan, S., M. Peng, and W.Wang. 2016. User access mode selection in fog computing based radio access networks. In IEEE International Conference on Communications (ICC),(May 2016),1-6.

59. Nazmudeen, M.S.H., A.T. Wan, and S.M. Buhari. 2016. Improved throughput for power line communication (plc) for smart meters using fog computing based data aggregation approach. In IEEE International Smart Cities Conference (ISC2), (Sept 2016), 1-4.

60. Hassan, M.A., M. Xiao, Q. Wei, and S.Chen. 2015. Help your mobile applications with fog computing. In 12th Annual IEEE International Conference on Sensing, Communication, and Networking Workshops (SECON Workshops), (June 2015), 1-6.

61. Deng, R., R. Lu, C. Lai, T.H. Luan, and H. Liang. 2016. Optimal workload allocation in fog cloud computing towards balanced delay and power consumption. IEEE Internet of Things Journal PP(99): 1-1.

62. Wang, Juan, and Di Li. "Task scheduling based on a hybrid heuristic algorithm for smart production line with fog computing." Sensors 19 , no. 5 (2019): 1023.

Published By:

Blue Eyes Intelligence Engineering \& Sciences Publication 


\section{AUTHORS PROFILE}

Harsimrat Singh, pursuing Master's of Technology in Computer Science \& Engineering from Guru Nanak Dev University Amritsar. He pursued Bachelor's of Technology in Computer Science \& Engineering from Amritsar College Of Engineering \& Technology, Manawala in year 2018. His research interest are Cloud Computing and Data Mining.

Chetan Marwaha, pursued Master of Computer Applications in Computer Science from Guru Nanak Dev University, Amritsar, India. He is working as System Manager in Department of Computer Science, Guru Nanak Dev University, Amritsar. He has published more than 10 research papers in UGC Approved Journals. His main research area focuses on Digital Image Processing, Image Segmentation, Web App. Development. He has 15 years of teaching experience. 